\title{
Fragility and compressibility at the glass transition
}

\author{
U. Buchenau* and A. Wischnewski \\ Institut für Festkörperforschung, Forschungszentrum Jülich, Postfach 1913, D-52425 Jülich, Germany \\ (Received 7 January 2004; revised manuscript received 22 March 2004; published 9 September 2004)
}

\begin{abstract}
Isothermal compressibilities and Brillouin sound velocities from the literature allow us to separate the compressibility at the glass transition into a high-frequency vibrational and a low-frequency relaxational part. Their ratio shows the linear fragility relation discovered by $\mathrm{x}$-ray Brillouin scattering, though the data bend away from the line at higher fragilities. Using the concept of constrained degrees of freedom, one can show that the vibrational part follows the fragility-independent Lindemann criterion; the fragility dependence seems to stem from the relaxational part. The physical meaning of this finding is discussed.
\end{abstract}

DOI: 10.1103/PhysRevB.70.092201

PACS number(s): 64.70.Pf, 77.22.Gm

Very recently, Scopigno et al. ${ }^{1}$ compiled x-ray Brillouin data in glasses, comparing the integrated Brillouin line intensity to the intensity of the central line. They found a linear relation between this ratio and the fragility $m$ $=\partial \log \eta / \partial\left(T_{g} / T\right)$, defined in terms of the steep rise of the viscosity $\eta$ towards the glass temperature $T_{g}$ with decreasing temperature in the supercooled liquid.

This striking result poses two questions. The first is a more technical point: Does the momentum transfer range of the X-ray Brillouin technique still reflect the long-wavelength limit? The second is more fundamental: Is the fragility related to the Brillouin intensity or to the central line intensity? Scopigno et al. only demonstrate a linear relation to the ratio of these two quantities. The present paper intends to address these two questions by a comparison to literature data on the long-wavelength limit.

The total scattering of a supercooled liquid at lowmomentum transfer is given by its isothermal compressibility $\chi_{T}:$

$$
\lim _{Q \rightarrow 0} S(Q)=\rho \frac{k T}{M} \chi_{T},
$$

where $\rho$ is the density and $M$ is the average atomic mass. Equation (1) has been found to be valid for several molecular and polymeric supercooled liquids ${ }^{2}$ on the nm length scale of the $\mathrm{x}$-ray Brillouin technique.

The X-ray Brillouin experiment splits the total scattering $S(Q)$ into an apparently elastic central component $S_{I S}(Q)$ and two Brillouin lines of summed intensity $S_{\text {Brill }}(Q)$. The longitudinal sound velocity $v_{l \infty}$ at the Brillouin line defines a highfrequency Brillouin compressibility $\chi_{B r i l l}=1 / \rho v_{l \infty}^{2}$. The ratio $\alpha_{\text {scatt }}=S_{\text {Brill }}(Q) / S_{I S}(Q)$ at the glass temperature $T_{g}$ reported by Scopigno et al. ${ }^{1}$ should equal the ratio $\alpha_{\chi}$ between vibrational and relaxational compressibility:

$$
\alpha_{\chi}\left(T_{g}\right)=\frac{\chi_{\text {Brill }}\left(T_{g}\right)}{\chi_{T}\left(T_{g}\right)-\chi_{\text {Brill }}\left(T_{g}\right)} .
$$

Table I compiles literature data of the isothermal com- pressibility and the Brillouin sound velocity. Most of the Brillouin sound velocities in Table I were obtained by light scattering; at the glass transition, light and x-ray scattering sound velocities still agree. ${ }^{10,14,18}$

As shown in Fig. 1(a), the general tendency is the same and the three common cases of glycerol, OTP, and Se agree

TABLE I. Isothermal and Brillouin compressibility at the glass transition. Abbreviations: $\mathrm{PIB}=$ polyisobutylene, $\mathrm{PB}$ =polybutadiene, $\quad \mathrm{PET}=$ polyethylenteraphtalate, $\quad$ OTP $=$ orthoterphenyl, $\mathrm{CKN}=\mathrm{K}_{3} \mathrm{Ca}_{2}\left(\mathrm{NO}_{3}\right)_{7}, \quad \mathrm{PVAC}=$ polyvinylacetate, $\mathrm{BPA}-\mathrm{PC}=$ polycarbonate, $\mathrm{PS}=$ polystyrene, $\mathrm{PMMA}=$ polymethylmethacrylate, and $\mathrm{PVC}=$ polyvinylchloride.

\begin{tabular}{lcccccc}
\hline \hline Substance & $\begin{array}{c}T_{g} \\
(\mathrm{~K})\end{array}$ & $\begin{array}{c}\rho \\
\left(\mathrm{kg} / \mathrm{m}^{3}\right)\end{array}$ & $\begin{array}{c}v_{l \infty} \\
(\mathrm{m} / \mathrm{s})\end{array}$ & $\begin{array}{c}v_{t \infty} \\
(\mathrm{m} / \mathrm{s})\end{array}$ & $\begin{array}{c}\chi_{\text {Brill }} \\
\left(\mathrm{GPa}^{-1}\right)\end{array}$ & $\begin{array}{c}\chi_{T} \\
\left(\mathrm{GPa}^{-1}\right)\end{array}$ \\
\hline $\mathrm{BeF}_{2}$ & $598^{\mathrm{a}}$ & $1900^{\mathrm{c}}$ & $4570^{\mathrm{c}}$ & & 0.0252 & \\
$\mathrm{SiO}_{2}$ & $1450^{\mathrm{a}}$ & $2200^{\mathrm{d}}$ & $6480^{\mathrm{d}}$ & $3988^{\mathrm{d}}$ & 0.0108 & \\
$\mathrm{~B}_{2} \mathrm{O}_{3}$ & $550^{\mathrm{e}}$ & $1792^{\mathrm{e}}$ & $3600^{\mathrm{f}}$ & $1933^{\mathrm{f}}$ & 0.0431 & $0.39^{\mathrm{e}}$ \\
$\mathrm{PIB}$ & $201^{\mathrm{b}}$ & $939^{\mathrm{g}}$ & $2994^{\mathrm{h}}$ & & 0.119 & \\
$\mathrm{Glycerol}$ & $187^{\mathrm{i}}$ & $1332^{\mathrm{i}}$ & $3583^{\mathrm{i}}$ & $1858^{\mathrm{j}}$ & 0.0586 & $0.287^{\mathrm{k}}$ \\
Salol & $218^{\mathrm{a}}$ & $1268^{\mathrm{l}}$ & $2382^{\mathrm{l}}$ & & 0.139 & \\
$1,4-\mathrm{PB}$ & $180^{\mathrm{a}}$ & $940^{\mathrm{g}}$ & $2500^{\mathrm{m}}$ & & 0.170 & \\
PET & $342^{\mathrm{a}}$ & $1350^{\mathrm{g}}$ & $2309^{\mathrm{n}}$ & & 0.139 & $0.324^{\mathrm{o}}$ \\
OTP & $241^{\mathrm{a}}$ & $1124^{\mathrm{p}}$ & $2550^{\mathrm{q}}$ & & 0.137 & $0.39^{\mathrm{p}}$ \\
Se & $308^{\mathrm{a}}$ & $4262^{\mathrm{r}}$ & $2000^{\mathrm{s}}$ & & 0.0587 & $0.16^{\mathrm{r}}$ \\
$\mathrm{CKN}$ & $343^{\mathrm{b}}$ & $2186^{\mathrm{e}}$ & $3190^{\mathrm{f}}$ & $1497^{\mathrm{f}}$ & 0.0450 & $0.132^{\mathrm{e}}$ \\
PVAC & $304^{\mathrm{e}}$ & $1186^{\mathrm{e}}$ & $2492^{\mathrm{t}}$ & & 0.136 & $0.498^{\mathrm{e}}$ \\
BPA-PC & $418^{\mathrm{b}}$ & $1180^{\mathrm{u}}$ & $2176^{\mathrm{v}}$ & $938^{\mathrm{v}}$ & 0.179 & $0.511^{\mathrm{o}}$ \\
PS & $375^{\mathrm{b}}$ & $1028^{\mathrm{u}}$ & $2219^{\mathrm{w}}$ & & 0.198 & $0.558^{\mathrm{o}}$ \\
PMMA & $379^{\mathrm{b}}$ & $1161^{\mathrm{u}}$ & $2500^{\mathrm{x}}$ & $1278^{\mathrm{w}}$ & 0.138 & $0.473^{\mathrm{o}}$ \\
PVC & $347^{\mathrm{x}}$ & $1372^{\mathrm{u}}$ & $2198^{\mathrm{x}}$ & & 0.151 & $0.385^{\circ}$ \\
\hline \hline
\end{tabular}

aReference 1. $\quad{ }^{\mathrm{i}}$ Reference $10 . \quad$ qReference 18.

${ }^{\mathrm{b}}$ Reference 3. $\quad$ jReference $11 . \quad$ rReference 19.

${ }^{\mathrm{c}}$ Reference 4. $\quad{ }^{\mathrm{k}}$ Reference $12 . \quad{ }^{\text {s }}$ Reference 20.

${ }^{\mathrm{d}}$ Reference 5. $\quad{ }^{\mathrm{l}}$ Reference $13 . \quad{ }^{\mathrm{t}}$ Reference 21.

'e Reference 6. $\quad$ meference $14 . \quad$ uReference 22.

fReference 7. $\quad{ }^{\mathrm{n}}$ Reference $15 . \quad{ }^{\mathrm{V}}$ Reference 23.

gReference 8. $\quad{ }^{\circ}$ Reference $16 . \quad{ }^{\text {w}}$ Reference 24.

heference 9. $\quad$ ' $R$ Reference $17 . \quad$ Reference 25. 


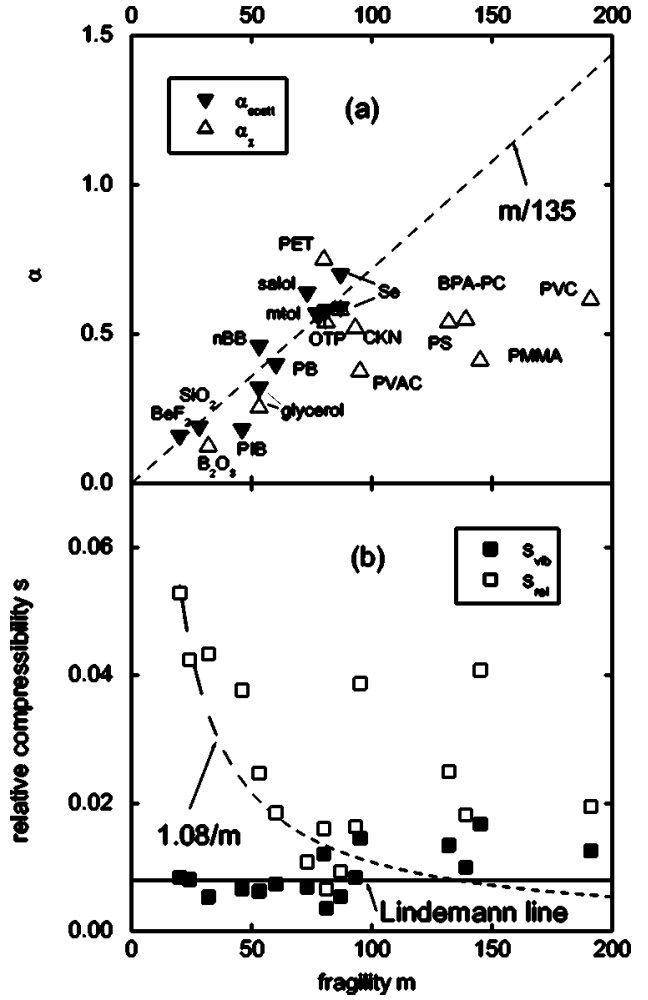

FIG. 1. (a) Comparison of $\alpha$ values from x-ray Brillouin scattering and from the long-wavelength limit. (b) Fragility dependence of normalized vibrational and relaxational compressibilities.

reasonably well in both sets of data. The literature data go to much higher fragility and thus reveal a bending away from the postulated line: at higher fragilities, the compressibility ratio becomes fragility independent. But even so, the answer to our first question is clear: the relation discovered by $\mathrm{x}$-ray Brillouin scattering ${ }^{1}$ is a true property of the longwavelength limit.

In order to answer the second question-namely, whether the Brillouin line or the central line provides the relation to the fragility - one needs to compare the vibrational or relaxational compressibility of different glass formers. This requires an appropriate normalization of the compressibility. It is reasonable to define a dimensionless ratio between the thermal energy at the glass transition and the vibrational and relaxational compression energy, respectively, by

$$
s_{v i b}=\frac{k_{B} T_{g} \chi_{\text {Brill }}}{v}, \quad s_{\text {rel }}=\frac{k_{B} T_{g}\left(\chi_{T}-\chi_{\text {Brill }}\right)}{v},
$$

where $v$ is an appropriate microscopic volume.

A priori, one would choose for $v$ the atomic volume. However, this choice is not justified, because glass formers are complex solids, with a mixture of strong and weak nearest-neighbor bonds. ${ }^{26,27}$ For instance, a polymer owes its material properties to a mixture of covalent and van der Waals bonds, very different in strength. Therefore one must take this difference in bonding strength into account.

Thus we define $v=v_{a t} / f_{s}$, where $v_{a t}$ is the atomic volume and $f_{s}$ is the fraction of soft degrees of freedom in the substance. The $f_{s}$ values in Table II were calculated assuming
TABLE II. Ratio of vibrational and relaxational compressibility at the glass transition.

\begin{tabular}{lcccccc}
\hline \hline Substance & $\alpha_{\chi}$ & $\alpha_{\text {scatt }}$ & $f_{s}$ & $M\left(10^{-27} \mathrm{~kg}\right)$ & $B / B_{0}$ & $m$ \\
\hline $\mathrm{BeF}_{2}$ & & $0.16^{\mathrm{a}}$ & $5 / 9$ & 26.0 & & $20^{\mathrm{a}}$ \\
$\mathrm{SiO}_{2}$ & & $0.191^{\mathrm{a}}$ & $5 / 9$ & 33.25 & & $24^{\mathrm{b}}$ \\
$\mathrm{B}_{2} \mathrm{O}_{3}$ & 0.124 & & $1 / 5$ & 21.92 & 5.6 & $32^{\mathrm{b}}$ \\
$\mathrm{PIB}$ & & $0.182^{\mathrm{c}}$ & $1 / 6$ & 7.75 & & $46^{\mathrm{b}}$ \\
$\mathrm{Glycerol}$ & 0.253 & $0.32^{\mathrm{a}}$ & $1 / 3$ & 10.91 & 3.1 & $53^{\mathrm{a}}$ \\
Salol & & $0.64^{\mathrm{a}}$ & $7 / 39$ & 13.66 & & $73^{\mathrm{b}}$ \\
$1,4-\mathrm{PB}$ & & $0.40^{\mathrm{a}}$ & $1 / 6$ & 8.96 & & $60^{\mathrm{a}}$ \\
$\mathrm{PET}$ & 0.751 & & $13 / 66$ & 14.49 & & $80^{\mathrm{d}}$ \\
OTP & 0.540 & $0.58^{\mathrm{a}}$ & $1 / 12$ & 11.93 & & $81^{\mathrm{b}}$ \\
$\mathrm{Se}$ & 0.579 & $0.7^{\mathrm{a}}$ & $2 / 3$ & 131.1 & & $87^{\mathrm{b}}$ \\
$\mathrm{CKN}$ & 0.516 & & $19 / 33$ & 31.76 & 2.1 & $93^{\mathrm{b}}$ \\
$\mathrm{PVAC}$ & 0.375 & & $1 / 4$ & 11.9 & & $95^{\mathrm{b}}$ \\
$\mathrm{BPA}-\mathrm{PC}$ & 0.539 & & $14 / 99$ & 12.78 & 2.1 & $132^{\mathrm{b}}$ \\
PS & 0.548 & & $5 / 48$ & 10.97 & & $139^{\mathrm{b}}$ \\
PMMA & 0.411 & & $2 / 9$ & 11.07 & 2.2 & $145^{\mathrm{b}}$ \\
PVC & 0.644 & & $4 / 21$ & 15.05 & & $191^{\mathrm{b}}$ \\
\hline \hline
\end{tabular}

${ }^{a}$ Reference 1 .

${ }^{\mathrm{b}}$ Reference 3 .

${ }^{\mathrm{c}}$ Reference 28.

${ }^{\mathrm{d}}$ Reference 29.

the stretching of all covalent bonds (including the Be-F bond) as well as the bond bending at boron, nitrogen, and carbon to be hard. All other degrees of freedom were considered to be soft. The resulting $s_{v i b}$ and $s_{r e l}$ values are shown in Fig. 1(b). Though the scatter of points is even worse than in Fig. 1(a), one observes that the fragility rise is only weakly correlated with the vibrational softening, but strongly with a decrease of the relaxational compressibility.

The first part of this result is consistent with empirical knowledge: The glass temperature tends to be about a factor of 0.6 smaller than the melting temperature $T_{m}$, which in turn follows the empirical Lindemann criterion. ${ }^{30}$ The Lindemann criterion states a mean-square vibrational displacement of the atoms in the crystal of $10 \%$ of the nearest-neighbor distance at the melting point. On the basis of a Debye model and a constant ratio of transverse and longitudinal sound velocity, one then expects the same vibrational compressibility in all glass formers at $T_{g}$, independent of the fragility $m$.

To quantify this Lindemann expectation, we assume a nearest-neighbor distance $d \approx v_{a t}^{1 / 3}$ and an average ratio of longitudinal to transverse sound velocity, $v_{100} / v_{t \infty} \approx 1.8$. The Lindemann criterion in the form improved by Gilvarry ${ }^{31}$ reads

$$
\left\langle u^{2}\right\rangle\left(T_{m}\right)=\frac{3 k_{B} T_{m}}{M \omega_{D}^{2}} \equiv(0.083 d)^{2} .
$$

Here $\left\langle u^{2}\right\rangle$ is the mean-square displacement in one direction and $\omega_{D}$ is the Debye frequency 


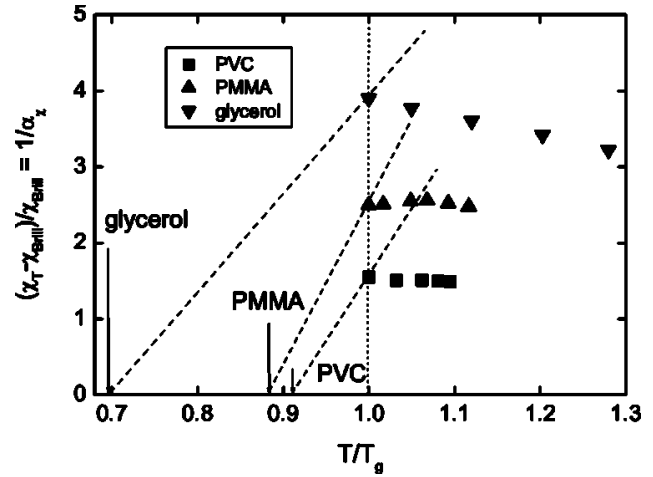

FIG. 2. Ratio of relaxational to vibrational compressibility for PVC, PMMA, and glycerol above $T_{g}$ (the references are the same as in Table I). The arrows mark the Vogel-Fulcher temperatures of the three glass formers; the dashed lines show the free volume expectation.

$$
\omega_{D}^{3}=\frac{18 \pi^{2}}{v_{a t}\left(1 / v_{l}^{3}+2 / v_{t}^{3}\right)} .
$$

With the above assumptions (including $T_{g} \approx 0.6 T_{m}$ ), one finds

$$
k_{B} T_{g} \approx 0.008 \frac{v_{a t}}{\chi_{\text {Brill }}} .
$$

This is the Lindemann line in Fig. 1(b), which is in reasonable agreement with the data points for the vibrational compressibility. Taking this Lindemann compressibility and the linear relation $\alpha=m / 135$ of Scopigno et al. [the dashed line in Fig. 1(a)], one gets $s_{\text {rel }}=1.08 / \mathrm{m}$, the dashed line in Fig. 1(b). We observe that this relation is only followed in the lower half of the fragility region, consistent with the bending away observed in Fig. 1(a).

The temperature dependence of the ratio between relaxational and vibrational compressibility $1 / \alpha_{\chi}$ is shown in Fig. 2 for three of the glass formers of Table I. There is no strong decrease of the ratio towards $T_{g}$, so the relaxational compressibility is proportional neither to the free volume nor to the excess entropy of the glass former. ${ }^{32}$ In the first case, it should extrapolate to zero at the Vogel-Fulcher temperature, in the second at the Kauzmann temperature (these two temperatures tend to lie close to each other ${ }^{33-35}$ ).
To clarify the physical meaning of the compressibility ratio, it is useful to rewrite it in terms of elastic moduli. Let $B$ and $G$ be the infinite-frequency bulk and shear modulus, respectively. $\chi_{T}=1 / B_{0}$, where $B_{0}$ is the zero-frequency bulk modulus (the zero-frequency shear modulus is zero above $\left.T_{g}\right) .1 / \chi_{\text {Brill }}=B+4 G / 3 \approx 1.7 B$, where we used again the approximation $v_{l \infty} / v_{t \infty} \approx 1.8$ mentioned in the derivation of the Lindemann line. Thus

$$
1 / \alpha_{\chi} \approx 1.7 \frac{B}{B_{0}}-1,
$$

so the ratio between relaxational and vibrational compressibility is a measure for the ratio between high-frequency and low-frequency bulk moduli. For strong glasses, this is high; for fragile glasses, it is low. In fact, for the five glasses where we know not only the isothermal compressibility, but also both the longitudinal and the transverse Brillouin sound velocity (see Table I), the calculated ratio $B / B_{0}$ in Table II decreases with increasing fragility (in those cases, it is possible to determine $B / B_{0}$ directly from experiment without any approximation).

The question is, what determines the ratio $B / B_{0}$ between long-time and short-time bulk moduli at the glass transition? This question can be translated into another question: What happens to the bulk modulus in the relaxation processes which bring the shear modulus down to zero? There are two extremes: (i) The bulk modulus is also brought down to zero. (ii) The bulk modulus is not affected at all. In the first case, $B / B_{0}$ is infinite and $\alpha_{\chi}=0$; in the second case, $B / B_{0}=1$ and $\alpha_{\chi} \approx 1.43$. However, these extremes are never reached; in Table II, $\alpha_{\chi}$ ranges from 0.124 to 0.751 , so $B / B_{0}$ ranges from 1.35 to 5.6 .

The consideration helps to understand the weakness of the temperature dependence in Fig. $2: B / B_{0}$ changes only slowly with temperature (if it changes at all). One also understands the physical meaning of the relation found by Scopigno et $a l .{ }^{1}$ : In strong glass formers, $B / B_{0}$ is large, in fragile ones small. Strong glass formers show strong relaxational density fluctuations on the scale of their vibrational compressibility, a factor of 3-4 stronger than fragile ones.

Helpful discussions with Giancarlo Ruocco and Reiner Zorn are gratefully acknowledged.
*Electronic address: buchenau-juelich@t-online.de

${ }^{1}$ T. Scopigno, G. Ruocco, F. Sette, and G. Monaco, Science 302, 849 (2003).

${ }^{2}$ E. W. Fischer and M. Dettenmaier, J. Non-Cryst. Solids 31, 181 (1978) and references therein.

${ }^{3}$ R. Böhmer, K. L. Ngai, C. A. Angell, and D. J. Plazek, J. Chem. Phys. 99, 4201 (1993).

${ }^{4}$ T. Scopigno, S. N. Yannopoulos, D. Th. Kastrissios, G. Monaco, E. Pontecorvo, G. Ruocco, and F. Sette, J. Chem. Phys. 118, 311 (2003).
${ }^{5}$ J. A. Bucaro and H. D. Dardy, J. Appl. Phys. 45, 5324 (1974).

${ }^{6}$ P. K. Gupta and C. T. Moynihan, J. Chem. Phys. 65, 4136 (1976).

${ }^{7}$ M. Grimsditch and L. M. Torell, in Dynamics of Disordered Materials, Springer Proceedings in Physics, Vol. 37, edited by D. Richter, A. J. Dianoux, W. Petry, and J. Texeira (SpringerVerlag, Berlin, 1989), p. 196; L. M. Torell and R. Aronsson, J. Chem. Phys. 78, 1121 (1983).

${ }^{8}$ D. W. van Krevelen, Properties of Polymers (Elsevier, Amsterdam, 1976), p. 70.

${ }^{9}$ A. P. Sokolov (private communication). 
${ }^{10}$ L. Comez, D. Fioretto, F. Scarponi, and G. Monaco, cond-mat/ 0305348 (unpublished).

${ }^{11}$ F. Scarponi, L. Comez, D. Fioretto, and L. Palmieri, Philos. Mag. 84, 1447 (2004).

${ }^{12}$ A. Gilchrist, J. E. Earley, and R. H. Cole, J. Chem. Phys. 26, 196 (1957).

${ }^{13}$ C. Dreyfus, M. J. Lebon, H. Z. Cummins, J. Toulouse, B. Bonello, and R. M. Pick, Phys. Rev. Lett. 69, 3666 (1992).

${ }^{14}$ D. Fioretto, U. Buchenau, L. Comez, A. Sokolov, C. Masciovecchio, A. Mermet, G. Ruocco, F. Sette, L. Willner, B. Frick, D. Richter, and L. Verdini, Phys. Rev. E 59, 4470 (1999).

${ }^{15}$ G. D. Patterson, J. Polym. Sci., Polym. Phys. Ed. 14, 1909 (1976).

${ }^{16}$ F. R. Schwarzl, Polymermechanik (Springer, Berlin, 1990), Table 12.1 .

${ }^{17}$ M. Naoki and S. Koeda, J. Phys. Chem. 93, 948 (1989).

${ }^{18}$ G. Monaco, D. Fioretto, L. Comez, and G. Ruocco, Phys. Rev. E 63, 061502 (2001).

${ }^{19}$ J. I. Berg and R. Simha, J. Non-Cryst. Solids 22, 1 (1976).

${ }^{20} \mathrm{G}$. Ruocco (private communication).

${ }^{21}$ J. K. Krüger, K. P. Bohn, R. Jimenez, and J. Schreiber, Colloid Polym. Sci. 274, 490 (1996).
${ }^{22}$ F. R. Schwarzl, Polymermechanik (Springer, Berlin, 1990), Abb. 6.12.

${ }^{23}$ G. D. Patterson, J. Polym. Sci., Polym. Phys. Ed. 14, 741 (1976).

${ }^{24}$ H. Krüger, in Optical Techniques to Characterize Polymer Systems, Studies in Polymer Science, Vol. 5, edited by H. Bässler (Elsevier, Amsterdam, 1989), p. 491.

${ }^{25}$ D. A. Jackson, H. T. A. Pentecost, and J. G. Powles, Mol. Phys. 23, 425 (1972).

${ }^{26}$ J. C. Phillips, J. Non-Cryst. Solids 34, 153 (1979).

${ }^{27}$ M. F. Thorpe, J. Non-Cryst. Solids 57, 355 (1983).

${ }^{28}$ B. Farago, A. Arbe, J. Colmenero, R. Faust, U. Buchenau, and D. Richter, Phys. Rev. E 65, 051803 (2002), obtain $S_{I S}(Q)=0.22$ in a neutron experiment; $\alpha_{\text {scatt }}$ follows from Eqs. (1) and (2).

${ }^{29}$ J. M. Saiter, E. Dargent, M. Kattan, C. Cabot, and J. Grenet, Polymer 44, 3995 (2003).

${ }^{30}$ F. A. Lindemann, Phys. Z. 11, 609 (1910).

${ }^{31}$ J. J. Gilvarry, Phys. Rev. 102, 308 (1956).

${ }^{32}$ J. Jäckle, Rep. Prog. Phys. 49, 171 (1986).

${ }^{33}$ C. A. Angell, J. Non-Cryst. Solids 131-133, 13 (1991).

${ }^{34}$ C. A. Angell, in Proceedings of the International School of Physics, "Enrico Fermi" Course CXXXIV, edited by F. Mallamace and H. E. Stanley (IOS Press, Amsterdam, 1997), p. 571.

${ }^{35}$ C. A. Angell, J. Res. Natl. Inst. Stand. Technol. 102, 171 (1997). 